\title{
Elementary proofs of the Liouville and Bôcher theorems for polyharmonic functions
}

\author{
by Ewa Ligocka (Warszawa)
}

\begin{abstract}
Elementary proofs of the Liouville and Bôcher theorems for polyharmonic functions are given. These proofs are on the calculus level and use only the basic knowledge of harmonic functions given in Axler, Bourdon and Ramey's book.
\end{abstract}

0. Introduction and preliminaries. The Liouville and Bôcher theorems are not a new subject. We found over 150 papers about the Liouville theorem for solutions of various (mostly elliptic) differential equations. The Bôcher theorem is somewhat less popular. Besides the classical case of harmonic functions, we found [4] and [5], where the Bôcher theorem was proved for solutions of elliptic equations ([4]) and some quasielliptic system of equations ([5]). There is also [3], where the Bôcher theorem for solutions of the Laplace-Beltrami equation was studied. While reading the excellent book of Axler, Bourdon and Ramey [2], we discovered that one can prove the Liouville and Bôcher theorems for polyharmonic functions, using the facts and ideas on harmonic functions given in that book. We must add only some elementary calculations. Our proofs will be on the calculus level.

We start with the following

0.1 . Definition. Let $\Omega$ be an open set in $\mathbb{R}^{n}$. A function $u: \Omega \rightarrow \mathbb{R}$ is $m$-polyharmonic iff $\Delta^{m} u=0$ on $\Omega$, where $\Delta^{m} u=\Delta(\Delta(\ldots \Delta u))$ ( $m$ times $)$, and $\Delta u=\sum_{j=1}^{n} \partial^{2} u / \partial x_{j}^{2}$ is the Laplace operator.

Let us next recall

0.2. Theorem (Almansi formula, see [1], Chapter V). If $u(x)$ is a function m-polyharmonic on the ball $B(0, R)$ then there exist functions $h_{0}, \ldots$ $\ldots, h_{m-1}$ harmonic on $B(0, R)$ such that

$$
u(x)=\sum_{k=0}^{m-1} h_{k}(x)|x|^{2 k} \quad\left(|x|=\left(\sum x_{i}^{2}\right)^{1 / 2}\right) .
$$

1991 Mathematics Subject Classification: Primary 31B30. 
In the sequel we shall prove the analogous formula for annular domains. We shall also use the Laurent series for harmonic functions (see [2], Chapter 10):

0.3. THEOREM If $h(x)$ is harmonic in an annular domain $A(R, r)=$ $\left\{x \in \mathbb{R}^{n}: r<|x|<R\right\}$ then

$$
h(x)= \begin{cases}\sum_{i=0}^{\infty} P_{i}(x)+\sum_{j=0}^{\infty} \frac{Q_{j}(x)}{|x|^{n+2 j-2}} & \text { for } n>2, \\ \sum_{i=0}^{\infty} P_{i}(x)+\sum_{j=1}^{\infty} \frac{Q_{j}(x)}{|x|^{n+2 j-2}}+c \ln |x| & \text { for } n=2 .\end{cases}
$$

Here $P_{i}$ and $Q_{j}$ are $i$-homogeneous and $j$-homogeneous harmonic polynomials, respectively, and the $i$-series converge for $|x|<R$, while the $j$-series for $|x|>r$.

1. The Liouville theorem. We start with the following.

1.1. THEOREM (Liouville theorem). Let $u$ be an m-polyharmonic function on $\mathbb{R}^{n}$. Assume that there exists $R \geq 0$ such that $u(x)>0$ if $|x|>R$. Then

$$
u(x)=c|x|^{2 m-2}+\sum_{k=0}^{m-2} H_{2 m-2 k-2}(x)|x|^{2 k},
$$

where $H_{2 m-2 k-2}$ is a harmonic polynomial of degree at most $2 m-2 k-2$. The leading homogeneous term of $u$ is equal to $c|x|^{2 m-2}+\sum_{k=0}^{m-2} Q_{2 m-2 k-2}(x)|x|^{2 k}$ where $Q_{2 m-2 k-2}$ is a $(2 m-2 k-2)$-homogeneous harmonic polynomial.

Proof. The proof will follow the simple idea of Nelson [7], which was also used in [2].

Let $u$ be as above. By Theorem $0.2, u(x)=\sum_{k=0}^{m-1} h_{k}(x)|x|^{2 k}$, where $h_{k}(x)$ is harmonic on $\mathbb{R}^{n}$ for each $k$.

Assume that $|x|>R$ and put $h(y)=\sum_{k=0}^{m-1} h_{k}(y)|3 x|^{2 k}$. Note that $h(y)$ is harmonic in $y$ and $h(y)=u(y)$ if $|y|=|3 x|$. Thus $h(y)>0$ on $\partial B(0,|3 x|)$ and by the maximum principle $h(y)>0$ on $B(0,|3 x|)$. By the mean value property we have

$$
\begin{aligned}
h(x)-h(0)= & \frac{1}{\operatorname{vol} B(x, 2|x|)} \int_{B(x, 2|x|)} h(y) d V_{y} \\
& -\frac{1}{\operatorname{vol} B(0,2|x|)} \int_{B(0,2|x|)} h(y) d V_{y} .
\end{aligned}
$$

Since $h$ is positive on $B(0,3|x|)$, we have 


$$
\begin{aligned}
|h(x)-h(0)| & \leq \frac{1}{\operatorname{vol} B(0,2|x|)} \int_{(B(x, 2|x|) \cup B(0,2|x|)) \backslash(B(x, 2|x|) \cap B(0,2|x|))} h(y) d V_{y} \\
& \leq \frac{1}{\operatorname{vol} B(0,2|x|)} \int_{B(0,3|x|) \backslash B(0,|x|)} h(y) d V_{y} \\
& \leq \frac{(3|x|)^{n}-|x|^{n}}{|x|^{n}} h(0)=\left(3^{n}-1\right) h(0) .
\end{aligned}
$$

Thus we get the inequality

$$
\left.\left|\sum_{k=0}^{m-1} h_{k}(x)\right| 3 x\right|^{2 k}-\left.\sum_{k=0}^{m-1} h_{k}(0)|3 x|^{2 k}\left|\leq\left(3^{n}-1\right) \sum_{k=0}^{m-1} h_{k}(0)\right| 3 x\right|^{2 x} .
$$

This inequality is valid for each $x \in \mathbb{R}^{n}$ with $|x|>R$. Hence there exists $c>0$ such that

$$
\left.\left.\left|\sum_{k=0}^{m-1} h_{k}(x)\right| 3 x\right|^{2 k}|<c| x\right|^{2 m-2} \quad \text { for }|x|>R .
$$

Let $R_{1}>R$. By the Cauchy inequalities for harmonic functions we have, for each multiindex $\alpha$,

$$
\left|\sum_{k=0}^{m-1} D^{\alpha} h_{k}(0) 3^{2 k} R_{1}^{2 k}\right| \leq \frac{c R_{1}^{2 m-2}}{R_{1}^{|\alpha|}} .
$$

This implies that each $h_{k}(x)$ must be a harmonic polynomial of degree at most $2 m-2 k-2$. In particular, $h_{m-1} \equiv$ const.

\section{Polyharmonic functions on annular domains}

2.1. TheOREM. Let $u(x)$ be an m-polyharmonic function on an annular domain $A(R, r)=\{x: R>|x|>r\}$. There exist harmonic functions $h_{k}$, $k=0, \ldots, m-1$, on $A(R, r)$ and $j$-homogeneous harmonic polynomials $Q_{\mu, j}$ on $\mathbb{R}^{n}$ for $n / 2 \leq \mu \leq m$ and $0 \leq j \leq \mu-n / 2$ such that

$$
u(x)=\sum_{k=0}^{m-1} h_{k}(x)|x|^{2 k}+\sum_{m \geq \mu \geq n / 2} \sum_{0 \leq j \leq \mu-n / 2} Q_{\mu, j}(x)|x|^{2 \mu-2 j-n} \ln |x| .
$$

If $n$ is odd, or if $n$ is even and $n>2 m$, then the logarithmic term does not occur.

Pro of. Let us state the following technical

Lemma. Let $Q_{i}$ be an $i$-homogeneous harmonic polynomial. Then

$$
\begin{aligned}
& \Delta\left(Q_{i}|x|^{k}\right)=Q_{i}|x|^{k-2}(n+k-2+2 i) k, \\
& \Delta\left(Q_{i}|x|^{k} \ln |x|\right) \\
& =Q_{i}|x|^{k-2}[(n+k-2+2 i) k \ln |x|+(n+2 k-2+2 i)] .
\end{aligned}
$$


Hence

$$
Q_{i}=\Delta^{p}\left(Q_{i}|x|^{2 p}\right) \frac{1}{2^{p} p ! \prod_{l=1}^{p}(n+2 l-2+2 i)}=\Delta^{p}\left(c(n, p, i) Q_{i}|x|^{2 p}\right),
$$

(4) $\frac{Q_{i}}{|x|^{s}}=\Delta^{p}\left(\frac{Q_{i}}{|x|^{s-2 p}}\right) \frac{1}{\prod_{l=1}^{p}(n+2 l-s-2+2 i)(2 l-s)}$

$$
=d(n, p, s, i) \Delta^{p}\left(\frac{Q_{i}}{|x|^{s-2 p}}\right)
$$

if $s$ is odd or if $s$ is even and $s>2 p$ (we also assume that the denominator does not vanish).

If $s$ is even and $2 p \geq s$ then there exist $a, b \in \mathbb{R}$ such that

$$
\frac{Q_{i}}{|x|^{s}}=\Delta^{p}\left(Q_{i}|x|^{2 p-s}(a \ln |x|+b)\right) .
$$

Pr o o f. (1)-(4) can be proved by direct calculations; (5) follows from (1) and (2).

We can now prove our theorem by induction on $m$. For $m=1$ our statement is obvious. Suppose that it is valid for $m-1$. Let $u$ be an $m$ polyharmonic function on $A(R, r)$. Then $\Delta^{m-1} u$ is a harmonic function on $A(R, r)$. We shall use Theorem 0.3 and expand $\Delta^{m-1} u$ in its Laurent series

$$
\Delta^{m-1} u=\sum_{i=0}^{\infty} P_{i}(x)+\sum_{j=0}^{\infty} \frac{Q_{j}(x)}{|x|^{n+2 j-2}} \quad \text { for } n>2
$$

or

$$
\Delta^{m-1} u=\sum_{i=0}^{\infty} P_{i}(x)+\sum_{j=0}^{\infty} \frac{Q_{j}(x)}{|x|^{n+2 j-2}}+c \ln |x| \quad \text { for } n=2 .
$$

Our Lemma implies that

$$
\begin{aligned}
\Delta^{m-1} u(x)= & \Delta^{m-1}\left[\sum_{i=0}^{\infty} P_{i}(x)|x|^{2 m-2} c(n, m-1, i)\right. \\
& \left.+\sum_{j=0}^{\infty} \frac{Q_{j}(x)}{|x|^{n+2 j-2 m}} d(n, m-1, n+2 j-2, j)\right]
\end{aligned}
$$

if $n$ is odd or $n$ is even and $n>2 m$. The series are almost uniformly convergent because the coefficients $\{c\}$ and $\{d\}$ are bounded as fuctions of $i$ and $j$ respectively. 
If $n$ is even and $2 m \geq n$ we have

$$
\begin{aligned}
\Delta^{m-1} u(x)= & \Delta^{m-1}\left[\sum_{i=0}^{\infty} P_{i}(x)|x|^{2 m-2} c(n, m-1, i)\right. \\
& +\sum_{j>m-n / 2} \frac{Q_{j}(x)}{|x|^{n+2 j-2 m}} d(n, m-1, n+2 j-2, j) \\
& \left.+\sum_{0 \leq j \leq m-n / 2} Q_{j}(x)|x|^{2 m-2 j-n}\left(a_{j} \ln |x|+b_{j}\right)\right] .
\end{aligned}
$$

The above formulae imply that $u=h_{m-1}|x|^{2 m-2}+u_{1}$ if $n$ is odd or $n$ is even and $n>2 m$, while

$$
u=h_{m-1}|x|^{2 m-2}+\sum_{0 \leq j \leq m-n / 2} Q_{j}|x|^{2 m-2 j-n} a_{j} \ln |x|+u_{1}
$$

in the other case. Here $h_{m-1}$ is harmonic on $A(R, r)$ and $u_{1}$ is $(m-1)$ polyharmonic on $A(R, r)$.

This proves our statement. (We write our formula for $n>2$, but for $n=2$ the proof is the same.)

We now prove the following.

2.2. TheOREM. Let $u$ be an m-polyharmonic function on an annular domain $A(R, r)=\{x: R>|x|>r\}$. The function has in $A(R, r)$ a Laurent expansion

$$
\begin{aligned}
u(x)= & \sum_{i=0}^{\infty} S_{i}(x)+\sum_{j=0} \frac{T_{j}(x)}{|x|^{n+2 j-2 m}} \quad \text { if } n \text { is odd or } n \text { is even and } n>2 m, \\
u(x)= & \sum_{i=0}^{\infty} S_{i}(x)+\sum_{j>m-n / 2} \frac{T_{j}(x)}{|x|^{n+2 j-2 m}} \\
& +\sum_{m \geq \mu \geq n / 2} \sum_{0 \leq j \leq \mu-n / 2} Q_{\mu, j}(x)|x|^{2 \mu-2 j-n} \ln |x|
\end{aligned}
$$

if $n$ is even and $2 m \geq n$.

Here

- $S_{i}(x)$ is an i-homogeneous m-polyharmonic polynomial,

- $T_{j}(x)$ is a $j$-homogeneous m-polyharmonic polynomial,

- $Q_{\mu, j}(x)$ is a j-homogeneous harmonic polynomial.

Pr o of. We expand each $h_{k}$ from Theorem 2.1 in a Laurent series (see Theorem 0.3). We obtain 


$$
u(x)=\sum_{k=0}^{m-1}|x|^{2 k}\left(\sum_{i=0}^{\infty} P_{k, i}(x)+\sum_{j=0}^{\infty} \frac{Q_{k, j}(x)}{|x|^{2 n+2 j-2}}\right)
$$

if $n$ is odd or if $n$ is even and $n>2 m$, and

$$
u(x)=\sum_{k=0}^{m-1}|x|^{2 k}\left(\sum_{i=0}^{\infty} P_{k, i}(x)+\sum_{j=0}^{\infty} \frac{Q_{k, j}(x)}{|x|^{2 n+2 j-2}}\right)+(\text { logarithmic terms })
$$

if $n$ is even and $2 m \geq n$.

We define $S_{l}(x)=\sum_{k=0}^{m-1} P_{k, l-2 k}(x)|x|^{2 k}(x)$ with $P_{k, i} \equiv 0$ if $i<0$. To find $T_{l}(x)$ we put $q=m-1-k$ and observe that

$$
\frac{|x|^{2 k} Q_{k, j}(x)}{|x|^{n+2 j-2}}=\frac{|x|^{2 q} Q_{k, j}(x)}{|x|^{n+4 q+2 j-2 m}} .
$$

Hence, if we define $T_{i}(x)=\sum_{k=0}^{m-1} Q_{k, l-2 q}(x)(x)|x|^{2 q}$ we get the required result. (We put $Q_{k, j} \equiv 0$ for $j<0$.)

2.3. REMARK. Laurent expansions for solutions of elliptic differential equations were studied in [8] and [6].

3. The $m$ th Kelvin transform. Let $u$ be a function defined in $A(R, r)$. The $m$ th Kelvin transform $K_{m}(u)$ is defined on $A(1 / r, 1 / R)$ by

$$
K_{m}(u)(x)=\frac{1}{|x|^{n-2 m}} u\left(\frac{x}{|x|^{2}}\right) .
$$

We have

3.1. TheOREM. The function $u$ is m-polyharmonic on $A(R, r)$ iff $K_{m}(u)$ is $m$-polyharmonic on $A(1 / r, 1 / R)$.

Proof. For $m=1$ Theorem 3.1 is classical (see [2]).

If

$$
u(x)=\sum_{k=0}^{m-1} h_{k}(x)|x|^{2 k}
$$

then

$$
\begin{aligned}
K_{m}(u)(x) & =\sum_{k=0}^{m-1} \frac{1}{|x|^{2 k}} \cdot \frac{1}{|x|^{n-2 m}} h_{k}\left(\frac{x}{|x|^{2}}\right) \\
& =\sum_{k=0}^{m-1}|x|^{2(m-1-k)} \frac{1}{|x|^{n-2}} h_{k}\left(\frac{x}{|x|^{2}}\right)=\sum_{k=0}^{m-1}|x|^{2(m-1-k)} K_{1}\left(h_{k}\right)(x) . \\
\text { If } u(x) & =Q_{\mu, j}(x)|x|^{2 \mu-2 j-n} \ln |x| \text { then } \\
K_{m}(u)(x) & =-Q_{\mu, j}(x) \frac{1}{|x|^{2 \mu-n}} \cdot \frac{1}{|x|^{n-2 m}} \ln |x|=-Q_{\mu, j}(x)|x|^{2 m-2 \mu} \ln |x| .
\end{aligned}
$$


By the Lemma from the proof of Theorem 2.1 we have

$$
\Delta^{m-\mu}\left(Q_{\mu, j}(x)|x|^{2 m-2 \mu} \ln |x|\right)=Q_{\mu, j}(x)(a \ln |x|+b)
$$

and

$$
\Delta^{n / 2+j-1}\left(Q_{\mu, j}(x)(a \ln |x|+b)\right)=c \frac{Q_{\mu, j}(x)}{|x|^{n+2 j-2}}
$$

is harmonic. In Theorem 2.1 we have $j \leq \mu-n / 2$ and hence

$$
\begin{aligned}
\Delta^{m}\left(Q_{\mu, j}(x)|x|^{2 m-2 \mu} \ln |x|\right) & =\Delta^{\mu} \Delta^{m-\mu}\left(Q_{\mu, j}(x)|x|^{2 m-2 \mu} \ln |x|\right) \\
& =\Delta^{\mu}\left(Q_{\mu, j}(x)(a \ln |x|+b)\right) \\
& =\Delta^{\mu-n / 2-j+1}\left(c \frac{Q_{\mu, j}(x)}{|x|^{n+2 j-2}}\right)=0 .
\end{aligned}
$$

Hence Theorem 3.1 is a direct consequence of Theorem 2.1 and the classical case $m=1$.

3.2. Remark. If $n=2 m$ then the $m$ th Kelvin transform is simply superposition with the inversion map $x \rightarrow x /|x|^{2}$. Hence superpositions with Möbius mappings preserve $m$-polyharmonicity in this case.

\section{The Bôcher theorem}

4.1. THEOREM (Bôcher theorem). Let $u$ be an m-polyharmonic function on a punctured unit ball $B(0,1) \backslash\{0\}$. Assume that $u>0$ on $B(0,1) \backslash\{0\}$. Then either $u$ has a removable singularity at 0 or a pole with highest singular term

$$
\frac{P_{2 m-2}(x)+c|x|^{2 m-2}}{|x|^{n+2 m-4}}
$$

where $P_{2 m-2}(x)$ is an $(m-1)$-polyharmonic, $(2 m-2)$-homogeneous polynomial.

Proof. By Theorem 2.2, $u$ can be expanded in a Laurent series on $B(0,1) \backslash\{0\}$.

1) If $n>2 m$ then

$$
u(x)=\sum_{i=0}^{\infty} T_{i}(x)+\sum_{j=0}^{\infty} \frac{S_{j}(x)}{|x|^{n+2 j-2 m}} .
$$

Put

$$
u_{1}(x)=T_{0}+\sum_{j=0}^{\infty} \frac{S_{j}(x)}{|x|^{n+2 j-2 m}} .
$$

2) If $n<2 m$ and $n$ is odd then

$$
u(x)=\sum_{i=0}^{\infty} T_{i}(x)+\sum_{j=0}^{[m-n / 2]} \frac{S_{j}(x)}{|x|^{n+2 j-2 m}}+\sum_{j>m-n / 2} \frac{S_{j}(x)}{|x|^{n+2 j-2 m}} .
$$


Put

3) If $n \leq 2 m$ and $n$ is even then

$$
u_{1}(x)=T_{0}+\sum_{j>m-n / 2} \frac{S_{j}(x)}{|x|^{n+2 j-2 m}} .
$$

$$
\begin{aligned}
u(x)= & \sum_{i=0}^{\infty} T_{i}(x)+\sum_{n / 2 \leq \mu \leq m} \sum_{0 \leq j \leq \mu-n / 2} Q_{\mu, j}(x)|x|^{2 \mu-2 j-n} \ln |x| \\
& +\sum_{j>m-n / 2} \frac{S_{j}(x)}{|x|^{n+2 j-2 m}} .
\end{aligned}
$$

Put

$$
u_{1}(x)=T_{0}+Q_{n / 2,0} \ln |x|+\sum_{j>m-n / 2} \frac{S_{j}(x)}{|x|^{n+2 j-2 m}} .
$$

( $S_{j}$ and $T_{i}$ are, as before, $j$-homogeneous and $i$-homogeneous $m$-polyharmonic polynomials.)

In every case $u(x)-u_{1}(x) \rightarrow 0$ as $x \rightarrow 0$. Hence there exists $r>0$ such that $u_{1}(x)>0$ on $B(0, r) \backslash\{0\}$. Let us apply the $m$ th Kelvin transform to $u_{1}(x)$. In the first case we have

$$
K_{m}\left(u_{1}\right)(x)=\frac{T_{0}}{|x|^{n-2 m}}+\sum_{j=0}^{\infty} S_{j}(x)
$$

(the last series converges for $|x|>1$ and thus for all $x \in \mathbb{R}^{n}$ ). Since in this case $T_{0} /|x|^{n-2 m} \rightarrow 0$ as $x \rightarrow \infty$, there exists $R>0$ such that $\sum_{j=0}^{\infty} S_{j}(x)>0$ for $|x|>R$. By the Liouville theorem (Theorem 1.1), $S_{j}(x) \equiv 0$ for $j>2 m-2$ and

$$
S_{m-2}(x)=c|x|^{2 m-2}+\sum_{k=0}^{m-2} Q_{2 m-2-2 k}(x)|x|^{2 k}
$$

where $c \geq 0$ and $Q_{2 m-2-2 k}$ is a $(2 m-2-2 k)$-homogeneous harmonic polynomial.

In the second case

$$
K_{m}\left(u_{1}\right)(x)=T_{0}|x|^{2 m-n}+\sum_{j>m-n / 2} S_{j}(x) .
$$

For large $|x|$ we have $\left|T_{0}\right| \cdot|x|^{2 m-2}<\left|T_{0}\right| \cdot|x|^{2 m-2}$. Hence we can apply the Liouville theorem to the function $\left|T_{0}\right| \cdot|x|^{2 m-2}+\sum_{j>m-n / 2} S_{j}(x)$ and get our assertion.

In the third case we have

$$
K_{m}\left(u_{1}\right)(x)=-Q_{n / 2,0} \ln |x|+\sum_{j>m-n / 2} S_{j}(x) .
$$


For large $|x|$ we have $\left|Q_{n / 2,0}\right| \ln |x|<c|x|^{2}$. We can again apply the Liouville theorem to the function $c|x|^{2}+\sum_{j>m-n / 2} S_{j}(x)$. If $m=1$ and $n=2$ then $2 m-2=m-n / 2=0$ and hence the singular part of the Laurent series can be equal to $c \ln |x|, c<0$.

\section{References}

[1] N. Aronszajn, T. Creese and L. Lipkin, Polyharmonic Functions, Clarendon Press, Oxford, 1983.

[2] S. Axler, P. Bourdon and W. Ramey, Harmonic Function Theory, Springer, 1992.

[3] B. R. Choe, Bôcher's theorem for M-harmonic functions, Houston J. Math. 18 (1992), 539-549.

[4] S. D. Eŭdel'man and T. G. Pletneva, Bôcher's theorem for positive solutions of elliptic equations of arbitrary order, Mat. Issled. 8 (1973), 173-177, 185 (in Russian).

[5] A. I. Firdman, The generalized Bôcher theorem for positive solutions of quasielliptic equations, Voronezh. Gos. Univ. Trudy Mat. Fak. Publ. 1973, 111-121; Ref. Zh. Mat. 1974 7B 331 (in Russian).

[6] R. Harvey and J. C. Polking, A Laurent expansion for solutions to elliptic equations, Trans. Amer. Math. Soc. 180 (1973), 407-413.

[7] E. Nelson, A proof of Liouville's theorem, Proc. Amer. Math. Soc. 12 (1961), 995.

[8] M. Wachman, Generalized Laurent series for singular solutions of elliptic partial differential equations, ibid. 15 (1964), 101-108.

Department of Mathematics, Informatics and Mechanics

Warsaw University

Banacha 2

02-097 Warszawa, Poland

E-mail: elig@hydra.mimuw.edu.pl 